\title{
9 DIENSTSTELLEN UND EINRICHTUNGEN DER NSDAP UND IHRER GLIEDERUNGEN UND VERBÄNDE
}

Als Ersatz für das in erheblichem Umfang verlorene Schriftgut der regionalen Dienststellen in Mittel- und Ostdeutschland und in den eingegliederten Gebieten können personenbezogene Unterlagen im Berliner Document Center in Betracht kommen; auf die Angaben dazu in Teil 1, S. 455, wird verwiesen.

\subsection{Reichsleitung der NSDAP}

In dem bei der ZDM in Moskau für Schriftgut der NSDAP gebildeten Sammelbestand 1409 befinden sich Rundschreiben und -erlasse sowie Aktensplitter aus dem Stab des Stellvertreters des Führers bzw. der Parteikanzlei (23 Bde, 1937-1945), der Reichspropagandaleitung (18 Bde, 1929-1945, u. a. betr. Juden und Freimaurer 1932-1937, Wirtschaftspolitischer Dienst) und der Reichsjugendführung (22 Bde, vor allem über Einsatz in den besetzten Ostgebieten 1943-1944, Betreuung von Umsiedlern, Meldung zum Wehrdienst, Zeitschriften). Aus dem Kolonialpolitischen Amt stammen Informationsblätter (1937-1945), Referate eines Kongresses in Belgien (6 Bde, 1940), der Entwurf eines Reichskolonialgesetzes (1941-1943) und Unterlagen über die Wirtschaft in Kamerun, Togo und Belgisch-Kongo (7 Bde, 1938-1939), aus dem Rassenpolitischen Amt lediglich Erlasse und Vorschriften (3 Bde, 1936-1944), vom Chef der Auslandsorganisation nur Korrespondenz (1937-1938) mit einzelnen Ortsgruppen und dem Reichsrechtsamt, von dem selbst Unterlagen für ein neues Strafrecht (1935-1945) vorliegen. Vom Beauftragten des Führers für die NSDAP in Österreich gelangten einige Aktensplitter in das BA KO (Best. R 45, 8 Bde); sie betreffen Mitgliedschaftsangelegenheiten (1928-1930, 1935-1938) und den „Österreichischen Pressedienst“ (1933-1934). In dem vom Ministerium für Staatssicherheit der ehemaligen DDR gebildeten Sammelbestand (BA DH) sind die Parteikanzlei (24 Bde, u. a. betr. Kirchensachen, NSKK, Ordensverleihungen, mit Eingaben und Anordnungen), das Hauptpersonalamt (13,9 Ifm Einzelfallakten und Karteien, u. a. betr. politische Beurteilungen), das Oberste Parteigericht (ca. 400 Bde Gnadensachen, 1933-1945) und verschiedene andere Ämter mit Akten (insgesamt ca. 40 Bde) u. a. über die Unterstützung „alter Kämpfer“, Parteistatistik, Verleihung von Kriegsverdienstkreuzen) vertreten. Größeren Umfang haben noch folgende Bestände:

\subsubsection{E Kanzlei des Führers der NSDAP}

ZDM MO, Best. 1355; BA DH, Best. EVZ

Überwiegend enthält der Moskauer Bestand (828 Bde, 1925-1945) Zuschriften aus der Bevölkerung mit Bitten um materielle Hilfe, Übernahme von Ehrenpatenschaften, Vermittlung eines Arbeitsplatzes, Gnadenerweise, Wiederaufnahme in die NSDAP und Glückwünsche zum Geburtstag mit Fotoalben und Gedichten (705 Bde, 1925-1944). Im übrigen sind vorhanden: Privatkorrespondenz Hitlers (8 Bde, 1932-1945), Runderlasse des Obersten SA-Führers (1931-1932) und -schreiben des Beauftragten für die 
Kinderlandverschickung (1938-1945), ein Protest von Ernsten Bibelforschern gegen die Judenverfolgung (1934), Unterlagen über die Verleihung der Sudetenmedaille (1939) und des Ritterkreuzes zum Eisernen Kreuz (1943-1944), Glückwünsche von Politikern und Diplomaten zum Geburtstag 1943, eine Liste und Beurteilungen von deutschen und inländischen Professoren in Spanien, Portugal und Südamerika (1944), einzelne eingereichte Manuskripte (8 Bde, 1938-1945), Unterlagen über das Familienwappen Hitlers (1935), Kunst und Architektur (4 Bde), Empfänge (1939), Zutrittsberechtigte zum Sperrkreis I im Führerhauptquartier, eine Sammlung von Fotos (41 Bde, 1900-1943). Der Sammelbestand 519 enthält eine Kartei mit spezieller Erlaubnis Hitlers aufgenommener Parteimitglieder (Anfangsbuchstaben P, Sch-W, 5 Bde mit ca. 250 Fällen, 1935-1944) und einzelne Akten über u. a. Volkssturm, das Verfahren gegen den Redakteur einer Parteizeitung, Frauenarbeitseinsatz.

Im BA DH sind neben einer Sammlung von Todesurteilen aus dem Amt für Gnadensachen (3 Bde, 1944-1945) ca. 30000 Patientenakten von Heil- und Pflegeanstalten vorhanden, die sich bis 1989 im Gewahrsam des DDR-Staatssicherheitsdienstes befanden und vermutlich bei der Außenstelle T 4 der Kanzlei gesammelt worden sind, die für die Ermordung der Geisteskranken verantwortlich war. Sie stammen von über 100 Provenienzstellen, von denen folgende Heil- und Pflegeanstalten mit jeweils mehr als 200 Einzelfallakten vertreten sind:

Andernach (ca. 280), Ansbach (ca. 500), Arnsdorf/Sachsen (ca. 1100), BedburgHau (ca. 340), Berlin-Buch, -Herzberge und-Wittenau (ca. 800), Eberswalde (ca. 700), Eglfing-Haar (ca. 250), Eichberg (ca. 500), Emmendingen (ca. 400), Erlangen (ca. 600), Graz-Feldhof (ca. 400), Groß-Schweidnitz (ca. 1100), Halberstadt (ca. 600), Herborn (ca. 300), Hochweitzschen (ca. 300), Hubertusburg (ca. 600), Jerichow (ca. 450), Kortau (ca. 340), Landsberg/Warthe (ca. 400), Leipzig-Dösen (ca. 340), Lübben (ca. 800), Meinkofen (ca. 500), Mauer-Oehling (ca. 280), Neuruppin (ca. 1300), Niedernhardt (ca. 400) Regensburg (ca. 400), Schkeuditz-Altscherbitz (ca. 450), Schönbrunn (ca. 400), Stadtroda (ca. 1600), Teupitz (ca. 700), Uchtspringe (ca. 1100), Ueckermünde (ca. 600), Weilmünster (ca. 250), Weinsberg (ca. 300), Wien-Am Steinhof und -Ybbs (ca. 600 bzw. 800), Wiesloch (ca. 560), Zschadraß (ca. 1000)

sowie aus den Justizvollzugsanstalten Brandenburg-Görden (ca. 1000) und Waldheim (ca. 800) und von den Gesundheitsämtern Bautzen (ca. 1000) und Gotha (ca. 1800).

Lit.: V. ROELCKE, G. HOHENDORF: Akten der „Euthanasie“-Aktion T 4 gefunden. 1993.

\subsubsection{E Kanzlei Rosenberg}

ZDM MO, in Best. 1409

Das im Bestand vereinigte Schriftgut aus den verschiedenen Kompetenzen Rosenbergs (93 Bde, 1933-1942) stammt wohl überwiegend aus dem Außenpolitischen Amt mit Berichten über die Beziehungen zu und die Lage in einzelnen Ländern, vor allem Rumänien, sowie deutsche Minderheiten (ca. 40 Bde, darin auch nachrichtendienstliches Material), über die weißrussische, tatarische und kaukasische Emigration (11 Bde) und die Besetzung Dänemarks (1939-1940). Vorhanden sind ferner Listen, Lebensläufe und Personalakten von Mitarbeitern und Akten über die Untersuchung unerlaubter Auslandskontakte (2 Bde, 1934) und Kirchenfragen (3 Bde). Korrespondenz des Stabsleiters Stellrecht ist an das Rassenpolitische Amt und das Reichsamt für das Landvolk gerichtet (je 1 Bd, 1943-1945), solche des Amtsleiters Schickedanz betrifft Auslandsreisen und den Herzog von Kent (1939). 


\section{$9.2 \quad$ Gauleitungen}

Das von den zuletzt 22 Gauleitungen, die für das Gebiet außerhalb der späteren Bundesrepublik zuständig waren, überlieferte Schriftgut besteht überwiegend aus Personalunterlagen, die 1945 von Besatzungstruppen sichergestellt wurden; dabei kann es sich zum Teil um Akten von Kreis- und Ortsgruppenleitungen handeln. Ersatz- und Ergänzungsüberlieferung ist - abgesehen von Akten der Gauleiter und Reichsstatthalter der Reichsgaue oben im Abschnitt 1.1 - vor allem zur Frühgeschichte der Partei in nennenswertem Umfang aus dem Hauptarchiv der NSDAP (BA KO, Best. NS 26) nur für die Gaue Berlin, Mark Brandenburg (und dem vorangehenden Gau Ostmark), Ostpreußen und Pommern vorhanden. Auf Drucksachen von Gauleitungen, die sich in der Deutschen Bibliothek Leipzig, im BA KO und im IfZ befinden, wird zu den einzelnen Gauen nur verwiesen, wenn sie größeren Umfang oder besondere inhaltliche Bedeutung haben; sie werden künftig in einem Verzeichnis nachgewiesen, das im IfZ bearbeitet wird und ebenfalls in den Texten und Materialien zur Zeitgeschichte veröffentlicht werden soll. Weitere Bestände an Drucksachen sind in polnischen und österreichischen Bibliotheken zu vermuten.

Gauleitung Berlin

BA DH Außer den bereits in Teil 1, S. 479, aufgeführten Beständen sind vor allem Personalunterlagen (ca. 2500 Bde), aber auch Sachakten u. a. des Gauschulungsamtes (15 Bde, 1938), des Gaugerichts und Rundschreiben vorhanden. Ein Splitterbestand (P, Pr.Br.Rep.61) enthält lediglich die Stärkemeldungen des Volkssturms von Februar 1945 und Stimmungsberichte der Ortsgruppe Germania (Juni 1944-April 1945).

\section{Gauleitung Danzig-Westpreußen, Danzig}

DAN, Best. 261// Überliefert sind (insgesamt 51 Bde, 1936-1945) Richtlinien, Rundschreiben, Aufrufe (9 Bde, 1938-1945), Personalunterlagen mit Beurteilungen (19 Bde, 1939-1944), eine Kartei der Parteigenossen mit den Mitgliedsnummern 1-9708 (3 Bde, 1935-1944, in Best. 273/I), Personalkarten des Kreises Berent (7 Bde) und einzelne Akten über Kulturangelegenheiten, weltanschauliche Erziehung (7 Bde, 1938-1945), Dienststellenverwaltung mit Listen der Dienststellen (1937), der Stellen im Auffanglager Brösen und der Amtswalter. Dazu kommt eine Sammlung von 912 Fotografien und 4 Alben der NSDAP Danzig-Stadt (1943-1944).

Ergänzungsüberlieferung liegt vor in Akten der Kreisleitung Bromberg (BRO, 92 Bde, 1939-1944, von 5 Bden 2 Mikrofilme im BA P, Best. PL-165) und vor allem der Kreisgerichte Danzig (256 Bde, 1933-1944), Danzig-Langfuhr (1238 Bde, 1932-1945), Danzig-Oliva (77 Bde, 1933-1941, alle DAN) und Thorn (GKW, Best. 88, 25 Bde, 1942-1945), die auch Urteile von Strafgerichten wegen Umgangs mit Polen enthalten.

Als Ersatzüberlieferung vorhanden sind das Mitteilungs-, ab 1940 Verordnungsblatt, Jg. 2-9 ( 1938-1945) und der NS-Gaudienst (1942-1945, beide DBL) und die Schrift Gauleiter Forsters: Die Volkstumsfrage im Reichsgau Danzig-Westpreußen (o. J., IfZ), ferner Vortragsmaterialien des Gauschulungsamtes (21 Nrn, 1940-1943) und Schriften der Adolf-Hitler-Schule Schulungsburg Danzig-Jenkau (10 Nrn, 1935-1940, beide BA KO, Best. NSD 38). Nachkriegsberichte von Angehörigen der politischen Führungsschicht (383 Bde, BA KO, Best. Ost-Dok. 8) stammen u. a. von der Gaufrauenschaftsführerin, dem NSV-Gauamtsleiter und Kreisleitern. 
Gauleitung Halle-Merseburg, Halle

BA DH

Der Bestand (ca. 4,3 lfm, 1933-1945) enthält Personalakten, Mitgliederlisten und ein Verzeichnis von politischen Leitern unter dem Gauleiter Joachim Eggeling. Relativ gut überliefert ist die Produktion der Gaufilmstelle (BA Filmarchiv B). Die 41 Filme (19331939) berichten u. a. von Gau- und Kreisparteitagen und -appellen, Festveranstaltungen, Besuchen Görings, Besichtigungen durch Gauleiter Jordan, Teilnahme am Reichsparteitag, Aufmärschen der HJ und der DAF-Gauschule Raitzsch. Unter dem Titel „Ein Gau baut auf" wurde 1937 ein Tätigkeitsbericht veröffentlicht (DBL).

Gauleitung Kärnten, Klagenfurt

KLA

Lediglich aus Privatbesitz konnte Sammlungsgut erworben werden. Es enthält u. a. Teile der Fotosammlung des Gauarchivs mit über 1000 Fotos vor allem zur NS-Frauenschaft, NSV, WHW, HJ, von Besuchen prominenter Politiker und Parteiführer, der Umsiedlung der Südtiroler und Kanaltaler, Soldatenbetreuung, Festen und Feiern, der Beisetzung von Gauleiter Hubert Klausner, Baubeginn der Reichsautobahn. Sie werden ergänzt durch private Fotos vor allem der Folgen von Luftangriffen und vom Anschluß 1938. $\mathrm{Zu}$ den für die Sammlung gewonnenen amtlichen Akten verschiedenster Provenienz gehören solche über die Bombenangriffe auf Klagenfurt, zur Planung der Besetzung Jugoslawiens, zur Umbenennung von Straßen und über den NS-Gauverlag. Erlebnisberichte, Selbstbiographien, anderes biographisches Material und Interviews betreffen u. a. Anschluß 1938, Alltag im NS-Staat, Vermögensbeschlagnahme, Aussiedlung und Konzentrationslager, Klausner und Ernst Lerch, Adjutant von Gauleiter Odilo Globocnig. Ein Teilbestand (Sammlung Fritz) enthält politisches Propagandamaterial (Flugblätter, Streu- und Klebezettel, Plakate, Wahlkampfbroschüren), das vor allem nahezu lückenlos die Propaganda nach der Okkupation Österreichs und zur Anschluß-,,Volksabstimmung“ vom 10. April 1938 und durch Einzelstücke zur Tätigkeit von HJ und NSV, anläßlich der Reichspogromnacht 1938, zum Polenfeldzug und Lebensmittelbewirtschaftung dokumentiert. In Leipzig (DBL) befinden sich die „Parole des Monats“, Sonderausgaben des NS-Gaudienstes (1940-1945), Rednerdienst (1941) und Redner-Schnelldienst (1941-1942) sowie „Propagandadienst“ (1942) des Gaupropagandaamtes, eine Schrift über „Oberkrain“ (1942) und das Mitteilungs-, ab 1940 Verordnungsblatt der Gauleitung (1938-1944).

Gauleitung Magdeburg-Anhalt, Dessau

BA DH

Überliefert sind (insgesamt 10,22 lfm, 1933-1945, als NSDAP-Akten Sachsen-Anhalt weitere 2,2 lfm) sowohl Personal- als auch Sachakten und Unterlagen aus Verfahren vor dem Gaugericht. Eine Sammlung von 23 Druckschriften befindet sich in MD (Rep. G 15), „Die Parole“, Sonderausgabe Volkssturm 1945, in der DBL.

Gauleitung Mark Brandenburg (bis 1940 Kurmark), Berlin

BA DH

Der Bestand (36,4 Ifm als Gau Kurmark, weitere 4,15 lfm als NSDAP-Unterlagen Gau Brandenburg) enthält Personalunterlagen, Mitgliederlisten und Mitgliedsbücher, die z. T. auch von Kreisleitungen, so des Kreises Osthavelland, stammen. Der Moskauer NSDAP-Sammelbestand (ZDM MO, Best. 519) enthält lediglich Rundschreiben der Gauleitung an die Kassenverwalter (2 Bde, 1936-1944), in etwas größerem Umfang Akten der Kreisleitungen Forst und Sorau. Ein Bericht über den Gautag 1939 wurde veröffentlicht (DBL). 
Ergänzungsüberlieferung bieten Akten (P, in Pr.Br.Rep.55, ca. 40 Bde) des Provinzialverbandes Brandenburg über die Gauleitung, das Gaupersonalamt und andere Ämter mit Verordnungsblättern (1934-1939) und streng geheimen Gaubefehlen (1935), das Gaupresseamt, die Gaupropagandaleitung, über Kulturarbeit der SA (1938-1942). Außerdem sind Rundschreiben von Dienststellen der Partei aus den letzten Kriegsmonaten und Splitterakten aus Kreis- und Ortsgruppenleitungen vorhanden, die bei der Ortsgruppe Woltersdorf Rundschreiben der Gauleitung, Gaubefehle und Verordnungsblätter enthalten (3 Bde, 1930-1939).

Lit.: K. ADAMY, K. HÜBENER: Provinz Mark Brandenburg - Gau Kurmark. 1993.

\section{Gauleitung Mecklenburg, Schwerin}

Ihre Tätigkeit wird nur durch das Führer- und Amtsblatt (Jg. 1-11, 1935-1945), eine Festschrift zum Gautag 1935 und den Bericht „Werden und Sein eines Gaues“ (1938, alle DLB) dokumentiert.

\section{Gauleitung Niederdonau, Wien}

Die Überlieferung ist auf eine aus Privatbesitz erworbene Sammlung parteiinterner Anweisungen der Gauleitung (DÖW, 60 Blatt) und Rundschreiben des Amtes für Volkswohlfahrt (1942, BA KO, Best. NS 45) beschränkt; außerdem sind 35 Nrn des Mitteilungsblatts (1941-1944) und 12 von 78 erschienenen Heften der vom Gaupresseamt herausgegebenen Reihe "Niederdonau, Ahnengau des Führers“ vorhanden (BA KO, NSD 38).

Gauleitung Niederschlesien (1936-1940 Schlesien), Breslau GKW, Best. 825 Der Bestand (62 Bde, 1938-1941) enthält Personalakten (52 Bde, 1936-1944) und einzelne Akten über HJ (1939-1941), Personalverwaltung, Werbung für die WaffenSS, Büchersammlungen (3 Bde, 1938-1941), Wirtschaftsfragen (1941), der Moskauer NSDAP-Sammelbestand (ZDM MO, Best. 519) über Mobilmachungsvorbereitungen (1938-1939) und Rundschreiben des Gaupropagandaamtes (1941, 1944), auch Akten der Kreisleitung Sprottau.

Ersatzüberlieferung bieten Akten der Kreisleitungen Neumarkt (BR, 40 Bde, 19391944, von 33 Bden 5 Filme im BA P, Best. PL-045) und Reichenbach (BR, 2439 Bde, 1939-1944). Ferner gibt es den NS-Gaudienst „Die innere Front“ (Jg. 7-10, 1942 1944) und „Der Schanzer“. Niederschlesiens Kriegseinsatz-Zeitung (Nr. 1-69, 19441945, beide DBL).

Gauleitung Oberschlesien, Kattowitz

KAT; GKW, Best. 826

Der Kattowitzer Bestand (Teil früher OP, Best. 240, 2132 Bde, 1933-1944) stammt vor allem aus dem Gaupersonalamt und dem Gaustabsamt, wohl auch aus Gaupropagandaamt und Gaugericht, möglicherweise ebenfalls vom Gauwirtschaftsberater. Er umfaßt Organisationsanordnungen, Lageberichte, Besprechungsprotokolle, Anordnungen zur Deutschen Volksliste, Fragebögen, politische Beurteilungen, Akten über die Besetzung von Ämtern in Behörden und Positionen in der Industrie, Unterrichtsund Bibliotheksangelegenheiten, Kontrolle von Presse, Rundfunk und Film, Rassenpolitik, Maßnahmen in Industrie, Handel und Handwerk, aus der Parteigerichtsbarkeit und überwiegend Personalunterlagen einzelner Parteigenossen mit zahllosen gleichartigen Eingaben um Hilfe in Nationalitätenkonflikten (1941-1944). Politische und wirtschaftliche Lageberichte verschiedener Kreisleitungen (1941-1942) sowie Lageberichte der 
NS-Frauenschaft (1942) sowie Protokolle von Dienstbesprechungen über Wirtschaftsfragen und Arbeitskräfteprobleme wurden verfilmt (BA P, Best. PL-014, 4 Filme von 45 Bden).

In Warschau (insgesamt 236 Bde, 1940-1944) befinden sich aus dem Büro des Gauleiterstellvertreters Albert Hoffmann u. a. Unterlagen über organisatorische Fragen, Verbindungen der Polizei zu den Kreisleitungen (8 Bde, 1941-1942) und über einzelne SA-Mitglieder (8 Bde, 1940-1944), aus dem Gaustabsamt Korrespondenz mit den Kreisleitungen (2 Bde, 1943-1945), Beschwerden, Gesuche und Vorgänge über Vergehen (90 Bde, 1940-1945), vom Gaupersonalamt Korrespondenz in Personalangelegenheiten (3 Bde, 1940-1944), Beschwerden, Anträge, Beurteilungen (99 Bde, 1939-1944), vom Gauwirtschaftsberater Berichte über Landwirtschaft, Versorgung mit Lebensmitteln, Brennstoff, Land- und Bergbaumaschinen (7 Bde, 1941-1944) sowie einzelne Bände von Gauorganisations-, Gauschulungsamt, Gaupropagandaleitung, Gauamt fïr Volkstumsfragen und Gaupressedienst.

Gesondert überliefert ist ein Aktenrest (KAT, Best. 116/I-11, 3 Bde, 1944-1945) über Organisation und Ausbildung des Volkssturms, der durch entsprechende Unterlagen der Kreisleitung Rybnik (KAT, 2 Bde, 1945) ergänzt wird.

Von den meisten Kreisleitungen im Gau sind Akten und Aktenreste vorhanden, mehr als 10 Bände liegen jedoch nur vor von den Kreisleitungen Beuthen (67 Bde, 19331944), Bielitz (21 Bde, 1939-1944), Gleiwitz (98 Bde, 1935-1944), Hindenburg (20 673 Bde, 1933-1945, von 15 Bden 2 Filme BA P, Best. PL-015), Königshütte (185 Bde, 1939-1945, von 4 Bden 1 Film im BA P, Best. PL-016), Kattowitz (158 Bde, 19391945), Pleß (146 Bde, 1940-1945, u. a. Stimmungs- und Lageberichte, Unterlagen über Ermordung Angehöriger der polnischen Intelligenz 1943, über Lehrer, NS-Frauenschaft, Kreisparteigericht, von 71 Bden 4 Filme im BA P, Best. PL-141) und Rybnik (13 Bde, 1940-1945).

Lit.: K. FIEDOR: „Sukcesy“ gauleitera Wagnera czyli jak usuwano slady polskosci na Slasku. 1985. - K. GRÜNBERG: Nazi-Front Schlesien. Niemieckie organizacje polityczne w Wojewodztwie Slaskim w latach 1933-1939. 1963.

\section{Gauleitung Oberdonau, Linz}

Lediglich als Ersatzüberlieferung sind nachweisbar das Gauführungs-, ab 1940 Verordnungsblatt (Jg. 1-7, 1939-1945 DBL, teilweise auch IfZ und BA KO), die Tätigkeitsberichte „Oberdonau nach dem ersten und nach dem dritten Kriegsjahr“ (1940, 1942, DBL), Reden Gauleiter Eigrubers „Ein Gau wächst ins Reich“ (1941), das Mitteilungsblatt des Gaupropagandaamtes (1941) bzw. des Gaurings (1941-1942) und der NS-Gaudienst (1942-1945, alle DBL).

Gauleitung Ostpreußen, Königsberg GStA, Rep. 494 Aus dem Gauarchiv sind u. a. Rundschreiben, Gaubefehle, vertrauliche Informationen (3 Bde, 1926-1933, 1941-1943), Reste der Korrespondenz von Gauleiter Erich Koch (1928-1940), des Gaugeschäftsführers und des Gauschulungsleiters (je 1 Bd) und Akten über Kirchen (2 Bde, 1933-1938), Juden (1933-1934), verschiedene Organisationen (3 Bde, 1926-1944, u. a. über Artamanen) und folgender Teilprovenienzen überliefert: Gauorganisationsamt (3 Bde Korrespondenz und ca. 60 Einzelfallakten), NS-Betriebszellenorganisation (3 Bde, 1931-1937), Gaupropagandaleitung (2 Bde, 1932-1937), Sonderbeauftragter für die Wehrmacht (4 Bde Schriftwechsel) und (nur Aktensplitter) Gaupersonal-, -rechts-, grenzlandamt, -schatumeister und 
Amt für Agrarpolitik. Den Hauptteil des Bestandes bilden Akten über die einzelnen Gauinspektionen und Kreise (43 Bde), auch über die NSDAP im Memelland (3 Bde, 1930-1944).

Ergänzungsüberlieferung ist vorhanden von der Kreisleitung Schröttersburg (WA, 56 Bde, 1940-1945, 1 Film von 11 Bden im BA P (Best. PL-079). Veröffentlicht wurden die Festschriften und Tätigkeitsberichte „Das nationalsozialistische Ostpreußen“ (1934), „Wir bauen das neue Reich“ (1935), zum Gautag 1938 (alle DBL) und „Zehn Jahre Gau Ostpreußen“ (1943, IfZ), ferner die „Befehlsausgabe“ (Jg. 3-7, 1935-1939), Anregungen und Richtlinien für die Feiern zum 30. Januar, Erntedankfest 1938 und zur Sonnenwende und das Mitteilungsblatt des Gaurings für Volksaufklärung und Propaganda (1943-1944, alle DBL).

\section{Gauleitung Pommern, Stettin}

Der Hauptteil der überlieferten Akten befindet sich in Stettin (STE, Best. 312, 1652 Bde, 1934-1944), wurde jedoch wegen Zweifeln an der Archivwürdigkeit bisher nicht erschlossen. Ein Teilbestand in Warschau (GKW, Best. 784, 41 Bde, 1935-1944) enthält Akten über die einzelnen Kreisleitungen. Aus Akten des Gauschatzmeisters und des Gaukassenverwalters der NS-Frauenschaft stammt eine lückenhafte Sammlung von Rundschreiben und Anweisungen (GW, Rep.97 p, 8 Bände, 1937-1943). Vom Verordnungsblatt (bis 1940 „Der politische Leiter") sind die Jahrgänge 1934-1944 vorhanden, ferner der Bericht über das Gautreffen 1938 (beides DBL) und „Gau Pommern im Aufbau“ (1934, IfZ).

Lit.: A. CZARNIK: Ruch hitlerowski na Pomorza Zachodnim 1933-1939. 1969.

Gauleitung Sachsen, Dresden

BA DH

Der Bestand umfaBt Personalunterlagen (ca. 20000 Fälle) und wenige Sachakten. Im Moskauer NSDAP-Sammelbestand (ZDM MO, Best. 519) ist Korrespondenz der Gauleitung mit dem Hauptamt für Kommunalpolitik (1937) enthalten. Die Überlieferung in Dresden (DD) besteht aus Aktensplittern des Gauamts für Technik (1942-1944) und 34 Druckschriften (1924-1944), wird jedoch ergänzt durch Unterlagen des NS-Verlags fïr den Gau Sachsen (7,3 lfm, ca. 1900-1944). Es handelt sich um Zeitungsausschnitte über deutsche und ausländische Persönlichkeiten des öffentlichen Lebens und um Bilder von NS-Funktionären, Ritterkreuzträgern und anderen ausgezeichneten Soldaten, Sportlern, Künstlern und Diplomaten sowie einigen Wissenschaftlern (ca. 7125 Mappen). Nur Aktensplitter liegen vom Kreisgericht Dresden XI in Radeberg und von 2 Ortsgruppen vor. Als Drucksachen (DBL) sind überliefert Programme der "Sachsentreffen“, Gauappelle und -wochen (1934-1938), „Der Gau Sachsen“ (2. Aufl. 1939), „Kriegsdienst“ zur vertraulichen Unterrichtung der Hoheitsträger (1939-1944), Mitteilungen des Gaurings (Jg. 1-5, 1940-1945), der NS-Gaudienst (1936-1945) und ein Politischer Informationsdienst (Folge 3-11, 1944-1945).

\section{Gauleitung Salzburg}

Lediglich im BA KO (Best. NS 45) befinden sich Aktenreste mit Tätigkeitsberichten (u. a. „Land im Aufbau“), Reden des Gauleiters Rainer und Stellungnahmen des Gauamtes fïr Volksgesundheit zu einzelnen Anträgen nach dem Erbgesundheitsgesetz (5 Bde, 1938-1939, 1941-1944). Ergänzungsüberlieferung enthalten Akten aus dem Verfahren des Landgerichts Linz 1948 gegen den letzten Gauleiter Gustav Adolf Scheel (20 Blatt 
Kopien im DÖW). Nachweisbar ist ferner das Mitteilungsblatt der Gauleitung (Jg. 2-7, 1940-1945), der NS-Gaudienst (1938-1945, beide DBL) und ein Tätigkeitsbericht des Gauschulungsamtes (1941, BA KO, NSD 38).

Lit.: E. HANISCH: Nationalsozialistische Herrschaft in der Provinz. 1983.

\section{Gauleitung Steiermark, Graz}

Nachzuweisen sind als Ersatzüberlieferung (DBL) das Befehlsblatt (Jg. 1-7, 1939-1945), das Steiermark-Heft der Reihe: Die deutschen Gaue seit der Machtergreifung (1942), der NS-Gaudienst (1939-1945) und „Der Propagandist“ (1941-1943).

Der Bestand (202 Kts, 1938-1945) enthält wöchentliche Lageberichte der Gauleitung (1939-1942) und Lageberichte der Kreisleiter (14 Kts, 1939-1945) sowie einzelner Ortsgruppen (1941-1942), ferner der Kreiswirtschaftsberater (3 Kts, 1940-1941), der Industrie- und Handelskammern Eger, Reichenberg, Troppau (1939-1942) und der Regierungspräsidenten in Karlsbad und Troppau (1 Kt, 1942-1943). Aus dem Gaustabsamt ist des Nachrichtenblatt (1944-1945) überliefert, aus dem Gauorganisationsamt Korrespondenz mit der Reichsleitung (1941-1945), vertrauliche Weisungen und Informationen der Reichsleitung (1943-1944), Verfügungen des Gauleiters Konrad Henlein (1938-1945) und einzelner Abteilungen der Gauleitung (insgesamt 8 Kts, 1938-1945), Korrespondenz mit den Kreisorganisationsämtern (2 Kts, 1939-1940).

Aus dem Gaupersonalamt stammen u. a. Akten über den Verkehr mit den Kreisleitungen (16 Kts, 1938-1945), politische Beurteilungen, Personalverzeichnisse und Korrespondenz (40 Kts, 1939-1945), Listen von Angehörigen der Internationalen Brigaden in Spanien (1938-1944), der Opfer des sudetendeutschen Kampfes (1942) und Amtswalter in den Kreisen Tetschen und Saaz, Personalakten Henleins (1939-1944) und des Reichstreuhänders der Arbeit Dr. Fritz Köllner (1939-1944).

Nur mit wenigen Akten vertreten sind Gauschulungsamt (Berichte über Schulungen 1941-1944, Listen verhafteter Mitglieder der Sudetendeutschen Partei und des Sudetendeutschen Freikorps 1939), Gaupropagandaamt (Zeitschrift „Der Propagandist“ 1943-1944, Fernschreibberichte über Luftangriffe 1941-1942), Gaupresseamt (vertrauliche Berichte 1939-1941, Flugblätter), Gauamt für Volksgesundheit (Weisungen und Rundschreiben 1938-1941), Gauwirtschaftsberater (Zeitschrift „Der Gauwirtschaftsberater“ 1942-1943, einzelne Weisungen 1943), Gauamt für Volkstumsfragen (Weisungen, Rundschreiben, Statistiken 1938-1945, Unterlagen über Wohnungsfragen 1941, Transporte mit Fremdarbeitern 1942-1944) und Gauarchiv (1938-1944). Aus der Parteigerichtsbarkeit sind Akten über einzelne Verfahren vom Gaurechtsamt (3 Kts, 19401942) und vom Gaugericht (5 Kts, 1939-1945) überliefert.

Einen eigenen Bestand bilden die Akten des von Gauhauptmann Anton Kreissl geleiteten Gauamtes für Kommunalpolitik (44 Kts, 1938-1945) mit Listen der Gemeindevorsteher und Korrespondenz mit ihnen 1938-1945, unvollständigen Reihen von Rundschreiben und Anordnungen der Gauleitung (1940-1945), Listen der Kreis- und Ortsgruppenleitungen (1940-1945), Berichten über Tagungen der Kreisamtsleiter für Kommunalpolitik (1940-1943), Rundschreiben und Anordnungen an die Landräte (1939), Fragebögen über kirchliche Einrichtungen (1939), Lageberichten aus den Kreisen (1941, 1943), Korrespondenz mit Polizeidienststellen (1940-1945) und Unterlagen über die Organisation der Kommunalverwaltungen (17 Kts, 1939-1945). 
Ein Aktenrest des Gaugrenzlandamtes Troppau (4 Bde betr. Neutitschein) befindet sich im Archiv in Troppau, und die Akten der Sudetendeutschen Partei im Staatlichen Zentralarchiv Prag enthalten z. T. Nachakten der Gauleitung bis 1939, u. a. der Kanzlei Konrad Henlein und Korrespondenz von Karl Hermann Frank, aus dem Gaurechtsamt auch Anträge von Staatsanwaltschaften auf Bestrafung von Parteigenossen mit Urteilsabschriften (4 Bde, 1940-1944). Eine Sammlung von Beschlüssen von Kreisgerichten (1940-1941) gelangte nach Koblenz (BA KO, in Best. NS 45).

Akten von Kreisleitungen liegen in den regional zuständigen tschechischen Archiven; sie stammen u. a. von den Kreisleitungen Freudenthal, Dux, Gablonz, Leitmeritz, Brüx, Troppau, Sternberg, Aussig, Freiwaldau, Jägerndorf, Hohenstadt und Mährisch Schönberg.

An Druckschriften sind überliefert (DBL) „Der Propagandist“ (1-6, 1939-1944), ein Propagandadienst für Ortsgruppen und Redner (1944-1945), Mitteilungen des Gaurings (1939-1944) und ein Bericht des Gaupropagandaleiters „Von der SdP zur NSDAP“ (1939).

Gauleitung Thüringen, Weimar

BA DH; WE Vorhanden sind aus den Beständen der DDR-Staatssicherheit überwiegend Personalunterlagen, Karteikarten über Mitglieder und nur einzelne Sachakten (ca. 25 lfm, 19331945). Inhaltlich bedeutsamer ist der Weimarer Bestand (z. T. früher BA KO, Best. NS 20). Er enthält u. a. einen Rest von Handakten des Gauleiters Fritz Sauckel (19211932), allgemeine Organisationsunterlagen (1933-1943), das Führer- und Amtsblatt des Gaues „Die Pflicht“" (2 Bde, 1934/35 und 1938, vollständig DBL), eine Dienstrangliste der Politischen Leiter (Ende 1937), Organisations- und Geschäftsverteilungsplan (1936), Zeitungsausschnitte über Kundgebungen und Versammlungen der NSDAP (5 Bde, 1936-1938), das Protokoll über eine Sitzung der Kreisleiter, Gauamtsleiter und Formationsführer (13. 11. 1939), Einladungen zu Dienstbesprechungen nach München (1939-1942), Unterlagen über wirtschaftspolitische Schulungen und Propaganda (19371941), Bauten und Produktion der Wilhelm-Gustloff-Werke (1937-1942), Aufklärung der Bevölkerung über verschärfte Luftschutzmaßnahmen (1943-1944) und Aktensplitter verschiedener Ortsgruppen. In der Plakatsammlung befinden sich Sonderausgaben und Beilagen von Zeitungen, Flugschriften und Plakate der NSDAP und ihrer Organisationen. Den Gausonderdienst „Die innere Front“ (Jg. 7-10, 1940-1943) und Berichte über die Gautage 1934 und 1935 besitzt die DBL.

Im BA KO (Kl. Erw. 848, 11 Bde, 1920-1945) verblieb private und dienstliche Korrespondenz Sauckels nebst Unterlagen über seine Beförderungen in der SA (1934-1937), die Adolf-Hitler-Schule Blankenhain (1939-1944) und Abrechnungen der Gauleitung vor allem über Lieferungen und Leistungen des Hotels „Elephant“ (1937-1945).

Gesondert überliefert sind (WE) die Akten und Pläne aus dem

\section{Zweckverband der Bauten am Platz Adolf Hitlers („Gauforum“) in Weimar}

Die Akten (1936-1948) betreffen den Gesamtkomplex (35 Bde), die „Halle der Volksgemeinschaft“ (4 Bde), das Gebäude (Turmhaus) für Reichsstatthalterei und Gauleitung (5 Bde) und die Bauten für die DAF (32 Bde, Stadthaus) und für die Gliederungen (15 Bde, Arkadenhaus). Sie werden durch 1068 Zeichnungen und Pläne ergänzt.

Lit.: J. JOHN: Rüstungsindustrie und NSDAP-Organisation in Thüringen 1933 bis 1939. 1974. 
Gauleitung Tirol-Vorarlberg, Innsbruck

Der Bestand vereinigt Splitter von Akten der Gauleitung Westgau (1 Bd, 1931-1933) und einzelne Bände aus dem Büro des Gauleiters (bis Mai 1938 Edmund Christoph, dann Franz Hofer), des Gauorganisations-, -schulungs-, -personal- und -presseamtes (mit Presseausschnittsammlung, Propagandamaterial, Zeitungen, Zeitschriften, Broschüren aus dem Gaupressearchiv) und des Amtes für Kommunalpolitik (insgesamt 30 Bde, 1938-1943). Er enthält ferner Erhebungsbögen der Parteistatistik 1939 (35 Bde), Personalakten alter Kämpfer (8 Bde), Akten zur Verleihung der Erinnerungsmedaille 13. 3. 1938 (5 Bde), Personalakten von SS-Führern (3 Bde), Listen nationalsozialistischer Organisationen (2 Bde, 1938-1945) und der Ortsgruppen in Innsbruck (2 Bde, 19381939).

Ergänzungsüberlieferung bieten Akten aus den Verfahren des Landgerichts Innsbruck von 1948 gegen Hofer und 1949 gegen Dr. Hans Czermak, Leiter des Amtes für Volksgesundheit, wegen Überstellung von 700 Patienten aus Tiroler und Vorarlberger Anstalten in die Euthanasieanstalt Hartheim (30 bzw. 70 Blatt Kopien im DÖW). Gauverfügungsblatt (Ausgabe A, 1941-1944), NS-Gaudienst (1938-1945), Informationsblatt des Gaurings für Volksaufklärung (1940-1943) und Sammelrundschreiben des Gaupresseamtes (1938-1940) liegen in Leipzig vor (DBL).

Lit.: W. BEIMROHR: Staat und Partei des Dritten Reiches in Tirol. 1988. - H. SCHREIBER: Vom AnschluB zum Krieg. Tirol 1938/39. 1991.

Gauleitung Wartheland, Posen

GKW, Best. 377 und 737; POS Die beiden GKW-Bestände (insgesamt 139 Bde, 1940-1944) enthalten u. a. politische Beurteilungen des Gaupersonalamtes, Monatsberichte der Kreisleitungen (8 Bde, 19411944), u. a. Liste der evangelischen und katholischen Geistlichen deutscher Nationalität (1942), Rundschreiben (2 Bde, 1941-1943), Personalakten mit Fragebögen und Beurteilungen der Mitarbeiter (57 Bde, 1940-1943), Personalbögen der Gauinspektion (2 Bde, 1939-1944) und der HJ (7 Bde, 1940-1944), Listen einberufener NSDAPMitglieder (1940-1943), Aktenreste aus dem Amt für Beamte, dem Gauschulungsund -verwaltungsamt sowie dem Amt für Volkstumsfragen (4 Bde, 1940-1943). Ferner sind Akten der Parteischule II, Litzmannstadt und ihres Leiters J. Auerswald über Organisation, Zulassung, Lehrgangsleiter, Bewerbungen, Beurteilung der Lehrgänge vorhanden (16 Bde, 1940-1944). Zum Posener Bestand (490 Bde, Ergänzungen im NachlaB des Gauredners Hans Hohnfeldt, 48 Bde, 1923-1945) gehören Unterlagen zur Organisation mit Tätigkeits- und Lageberichten (1941-1944), Akten des Gauamtes für Volkstumspolitik zur Deutschen Volksliste und des Rassenpolitischen Amtes, Anweisungen und Rundschreiben des Gauschatzmeisters, Rundschreiben des Gaustabsamtes und Fragmente von Akten des Gaugerichts, überwiegend jedoch Personalunterlagen (457 Bde).

Mit allen Teilbeständen und einem weitern GKW-Bestand (Best. 800, 377 Bde) wurden Akten der Kreisleitungen vereinigt, u. a. aus Kolo, Konin, Lask, Lentschütz, Lissa, Ostrowo, Posen, Schieratz, Schneidemühl, Turek, und der Kreisgerichte Schneidemühl und Rawitsch. Die Reste der Akten der Kreisleitung Litzmannstadt befinden sich überwiegend in Lodz (56 Bde, 1940-1944).

Die Sammlungen des Westinstituts (Instytut Zachodni) in Posen enthalten Aktenreste des Gauamtes für Volkstumsfragen u. a. mit Denkschriften zur Umsiedlung der Polen, Urteilen des Obersten Prüfungshofes für Volkstumsfragen, einen Volkspolitischen Lagebericht des Reichsinnenministeriums (Mai-Juli 1940) und Drucksachen zur Ansiedlung 
der Schwarzmeer- und Wolhyniendeutschen, der NS-Gaudienst (Jg. 1-6, 1939-1945) und das Mitteilungsblatt der Gaupropagandaleitung „Propaganda“ (1943-1944) liegen in der DBL.

Lit.: K. POSPIESZALSKI: Niemiecka Lista Narodowa w „Kraju Warty“. 1949.

Gauleitung Wien

AdR, Best. 04R008/1

Als Gauakten (ca. $6500 \mathrm{Kts}, 1938-1945$ ) sind vor allem Einzelfallakten des Gaupersonalamtes (kleinerer Teilbestand mit Einzelpersonalakten auch im StLAW) vereinigt, die jedoch auch Personen aus dem gesamten ehemaligen österreichischen Bundesgebiet sowie aus den 1939 eingegliederten südböhmischen und südmährischen Kreisen, umgesiedelte Volksdeutsche aus Südosteuropa und der ganzen Welt, Südtiroler Optanten und zugezogene Reichsdeutsche betreffen. Vereinzelte „Sachakten“ beziehen sich auf ganze Personengruppen (oft Firmenbelegschaften) oder Institutionen. Enthalten sind Vorakten der Vaterländischen Front (politische Beurteilungen und Personalakten von „Amtswaltern"). Den Einzelfällen zugeordnet wurden vielfach Schriftstücke fremder Provenienz, vor allem des Gauschatzamtes, der Österreichischen Legion (auch Hilfswerk Nordwest), aus der „Asozialenkartei“ und der „Warnkartei“ (vor Regimegegnern).

Die Akten enthalten Parallelüberlieferungen zur NS-Wiedergutmachungs- und Vermittlungsstelle (oben 2.1.3.2) sowie zur Tätigkeit der für Berufsbeamte zuständigen Dienststelle des Staatskommissärs Dr. Otto Wächter, deren Schriftgut nicht überliefert ist, und zur Parteigerichtsbarkeit. In ihnen finden sich zahlreiche Ausfertigungen von Anklageschriften und Gerichtsurteilen aus dem Zeitraum von 1900 bis 1950, Unterlagen über die Aktion zur Befreiung politischer Häftlinge nach dem Anschluß, über den Deutschen Volkssturm. Zwar bilden die Aufnahmeanträge für die Parteimitgliedschaft den Grundstock der Überlieferung, hinzu kommen jedoch viele Akten, die anläßlich der Einholung einer politischen Beurteilung angelegt wurden, z. B. zur Aufnahme in die Reichsschriftumskammer und andere Berufsorganisationen, bei Ernennung zum Reserveoffizier oder zum Beamten. Sie können daher auch Nicht-Parteimitglieder, Juden und - selbst emigrierte - Gegner des NS-Regimes betreffen.

Die erhalten gebliebenen Sachakten der Gauleitung befinden sich bis auf Berichte über Luftangriffe (AdR, in Best. 08R450, 8 Bde, 1942-1945) in folgenden Beständen im Wiener Stadt- und Landesarchiv:

\section{Gauamt für Kommunalpolitik}

Nur wenige Faszikel enthalten eine Verordnungssammlung (1 Fasz., 1937-1944) und allgemeinen Schriftwechsel (4 Fasz., 1938-1945).

Gauamt für Rassenpolitik

Der Bestand umfaBt Schulungsunterlagen (1 Fasz., 1941-1944), Vorgänge über verschiedene Rechtsfragen wie Namensänderungen, Ehrenpatenschaften Hitlers oder Wohnungsangelegenheiten (1 Fasz., 1940-1944), Akten über prinzipielle Volkstumsfragen, Zusammenarbeit mit dem Reichsbund Deutsche Familie mit Rundschreiben, Mütterschulung (2 Fasz., 1939-1943), die Verleihung des Ehrenkreuzes der deutschen Mutter (2 Fasz., 1940-1943), Erhebungen über kinderreiche Familien und Bevölkerungspolitik (2 Fasz., 1939-1940), Hausgehilfinnen und über Haushaltsüberprüfungen (1 Fasz., 19411944), Erhebungen über ungesunde Kinder und Behinderte (1 Fasz., 1941-1945), über die Erfassung Asozialer (1 Fasz., 1941-1945), Angelegenheiten von Mischlingen, insbesondere Ehegenehmigungen, Juden und Mischlinge in der Wehrmacht, Umgang von Ariern mit Juden und Mischlingen (1 Fasz., 1941-1944) sowie Angelegenheiten von 
Ostarbeitern (2 Fasz. 1941-1944). Unvollständig erhalten sind Akten über Ehegenehmigungen für Parteigenossen (4 Fasz., 1944), Anzeigen und Bittgesuche (4 Fasz.) sowie andere Einzelfallakten ( $37 \mathrm{Kts}$ ) (von Unterlagen über sogenannte asoziale Familien ca. 1500 Blatt Kopien DÖW).

Mit dem Bestand vereinigt sind Aktenreste vom Reichsbund Deutsche Familie (RDF), u. a. der Landesleitung (1 Fasz., 1938-1941), Tagungsunterlagen, Propagandaund Schulungsmaterial (2 Fasz., 1938-1941), Rundschreiben des Reichsbundesleiters (1 Fasz., 1938-1944), allgemeiner Schriftwechsel (2 Fasz., 1938-1944), Akten über den Beitritt von Mitgliedern (1 Mappe) sowie andere Einzelfälle (2 Fasz., A-Z), vom Gausippenamt (oben S. 52) und vom Gaurechtsamt ausschließlich Unterlagen über Erteilung von Genehmigungen für Rechtsanwälte zur Vertretung von Juden sowie zur Bildung von Treuhandgesellschaften (4 Fasz.). Als Ergänzungsüberlieferung sind bruchstückhafte Unterlagen von Ortsgruppen und Kreisleitungen vorhanden, vorwiegend Einzelpersonalangelegenheiten und Korrespondenzen (StLAW, 15 Fasz.), Stimmungsberichte (1939) sowie Korrespondenz verschiedener Ortsgruppen mit der Gauleitung (u. a. über Verkauf beschlagnahmter jüdischer Güter), Mitgliederlisten, Rundschreiben (u. a. betr. Verleihung von Auszeichnungen, HJ und BdM, NSKK) und statistische Monatsberichte der Gauleitung Wien (DÖW, insges. ca. 1800 Blatt Originale aus Privatbesitz), ferner Mitteilungs-, ab 1940 Verordnungsblatt der Gauleitung (1938-1944), NS-Gaudienst (Jg. 1-7, 1938/39-1944/45) und Mitteilungsblatt des Gaurings (1942-1944, alle DBL).

Lit.: G. JADSCHITZ: NSDAP und „AnschluB“ in Wien 1938. 1978.

\subsection{Gliederungen der NSDAP}

\subsubsection{SA (Sturmabteilungen der NSDAP)}

\subsubsection{E Oberste SA-Führung}

BA DH, Best. ZA II

Aus der Dienststelle des Chefs des Ausbildungswesens (Chef AW) sind Personalunterlagen (ca. 15000 Bde, 1933-1936) überliefert. Der Moskauer Sammelbestand (ZDM, Best. 1212) enthält lediglich einzelne Erlasse des Obersten SA-Führers vor allem in Personalangelegenheiten (4 Bde, 1933-1935, 1938-1940).

\subsubsection{SA-Gruppenführungen}

Das von den 14 SA-Gruppen in Mittel- und Ostdeutschland und den eingegliederten Gebieten in die Archive gelangte Schriftgut besteht überwiegend aus Personalunterlagen, von denen ein großer Teil vermutlich von unterstellten Brigaden und Standarten stammt. Ein umfangreicher Sammelbestand (BA DH, 143,8 lfm) ist noch unerschlossen. Außerdem befinden sich dort Personalunterlagen der Gruppen Berlin-Brandenburg in zwei Sammelbeständen (zusammen ca. $180 \mathrm{lfm}$, dabei auch Gruppenbefehle und aus Berlin Fragebögen und eine Fotosammlung, weitere Befehle, 1935-1938, ZDM MO, Best. 1212), Ostmark (13 Bde, mit Stammrollen, 1933-1939), Pommern (4,3 lfm, 1933-1936), Thüringen (ca. 23 lfm, Befehle, 1939-1944, auch ZDM, Best.1212) und von mecklenburgischen Einheiten der Gruppe Hansa (0,75 lfm, 1933-1934).

Zur Überlieferung folgender Provenienzen sind weitere Angaben möglich: 
SA-Gruppe Ostland, Königsberg

GKW, Best. 884

Die Aktenreste (17 Bde, 1933-1944) enthalten Befehle (1933-1943) und das Verordnungsblatt (1935), Dienstpläne und Unterlagen über Personalangelegenheiten der Standarten 82, 151 (Bischofsburg) und 445 (13 Bde) sowie Akten über Ausbildung und Ausrüstung (2 Bde, 1935-1944).

SA-Gruppe Sachsen, Dresden

ZDM MO, Best.1212; BA DH In Moskau enthalten die Sachakten (265 Bde) Anschriften- und Personallisten, eine Statistik der Disziplinarmaßnahmen, Rundschreiben über Kommandierungen (44 Bde, 1936-1939), Beförderungsvorschläge (23 Bde, 1939-1945) und betreffen u. a. Tauglichkeitsvorschriften, das Hilfslager Sachsen, Dienst der Studenten, Marine- und Reiter-SA, Schulungskurse, Sportveranstaltungen, Werbung für die kasernierte Standarte „Feldherrnhalle" (9 Bde, 1936-1938) und Angelegenheiten der einzelnen Standarten in Sachsen. Nach Abgabe von 116 Personalakten an die damalige DDR sind noch nach Alphabet und Einheiten geordnete Einzel- und Sammelpersonalakten (1019 Bde) sowie Personalakten der Angehörigen des Hilfslagers Sachsen (7 Bde, bis 1937) und der im Osten Gefallenen (20 Bde, 1941-1945) vorhanden. In Dahlwitz-Hoppegarten befinden sich weitere Personalunterlagen und -listen (ca. 142 lfm, 1933-1945), Aktensplitter einzelner Einheiten auch in Dresden.

SA-Gruppe Schlesien, Breslau

GKW, Best. 831, 57; BRE, Best. 290 In beiden Warschauer Beständen (305 bzw. 89 Bde, 1933-1945) sind überwiegend Personalakten überliefert. Außerdem enthalten sie Befehle, ebenfalls vor allem in Personalangelegenheiten, insbesondere der Brigade 17 (Oppeln) und ihrer Standarten (3 Bde, 1933-1939), von der Standarte 62 (Cosel) auch Unterlagen über Werbung, Aufnahme von SA-Leuten in die NSDAP, Übernahme in die Wehrmacht (15 Bde, 1936-1944, mit Liste arbeitsloser SA-Männer), Ausbildung, Schulung und Sport (8 Bde, 1937-1944), Ausrüstung (3 Bde, 1933-1944), von der Standarte 63 (Oppeln, weitere 13 Bde OP) über Veranstaltungen und Aufmärsche (8 Bde, 1933-1941), Schulung und Ausbildung (15 Bde, 1933-1944), Kassensachen und Ausrüstung (15 Bde, 1933-1944).

Auch in Breslau (101 Bde, 1932-1945) bilden Personalakten den Hauptteil (94 Bde), neben denen nur Sammlungen von Befehlen und Organisationsunterlagen (1932-1945) vorhanden sind. Dieser Bestand ist fast vollständig verfilmt (BA P, Best. PL-025, 27 Filme), desgleichen (Best. PL-024 bzw. -048, 3 Filme) Akten der Einheiten in Lüben (55 Bde) und Neumarkt (12 Bde).

SA-Gruppe Sudeten, Reichenberg

Der Bestand (1,4 lfm, 1938-1945) enthält von der Obersten SA-Führung Befehle (19381944), Personalbefehle (1942-1944), Organisationsanordnungen (1939-1945), das Verordnungsblatt (1931-1943), vom Führer der Gruppe ebenfalls Personalbefehle (19381939), Wochen- und Tagesbefehle (1939-1944), Stabsbefehle (1941-1942), Verwaltungsbefehle (1938-1945), Stammrollen der unterstellten Einheiten, Personalakten, Beurteilungen von Lehrgangsteilnehmern an der SA-Schule Hirschberg und der Reichsschule der SA Dresden (1940-1941), Fernschreiben (1940).

Von den nachgeordneten 5 Brigaden und 49 Standarten sind nur Aktensplitter überliefert, u. a. von der Brigade 104, Troppau (0,3 lfm), und den Standarten in Freudenthal, Freiwaldau, Bärn, Olmütz, Sternberg, Jägerndorf (TRO, weitere Bestände in LT und Pilsen). 
Außerdem vorhanden sind Akten der

SA-Gruppenschule Sudeten 2, Groß-Glockersdorf

LT

Sie enthalten (24 Kts, 1938-1945) u. a. Befehle, Verordnungen, Rundschreiben, Meldungen über gefallene und ausgezeichnete SA-Männer (1939-1943), Schriftwechsel (19391943) und Unterlagen über wirtschaftliche Angelegenheiten (1939-1945).

SA-Gruppe Warthe, Posen

GKW, Best. 81, 386, 699

Die Bestände (insgesamt 1700 Bde) enthalten fast ausschlieBlich Personalakten und -unterlagen (dabei Liste der aus dem Reich nach Posen versetzten SA-Leute 1942), auch Stammrollen (3 Bde, 1940-1942), ferner Anordnungen und Befehle, Vorgänge über Organisation und Propaganda (7 Bde, 1936-1944). Von der Brigade 187, Litzmannstadt, sind Aktenreste über Organisations-, Personal-, Ausbildungs- und Gesundheitsfragen erhalten (LO, 5 Bde, 1940-1944).

\section{SA-Gruppe Weichsel, Danzig}

DAN, Best. 1288/I

Der Bestand (243 Bde, 1930-1945) enthält außer Personalunterlagen (195 Bde, 19391944, weitere 87 Einzelfallakten der Standarte 5, Danzig, GKW) Anordnungen und Befehle (7 Bde, 1933-1940) und einzelne Akten über Reichsparteitag, Vereidigung (2 Bde, 1934-1938), Ausbildung und Sport (5 Bde, 1933-1939), Sippenforschung (1937), Presse, Sportveranstaltungen, Korrespondenz mit der SS (15 Bde, 1933-1939), Stärkemeldungen, Beförderungen, Aufnahme in die SA (10 Bde, 1932-1942), die Standarte 152 (Marienburg, mit Liste der Wehrmannschaft, 3 Bde, 1939-1943), Volkssturm (19441945) und den Aktenplan (1940).

\subsubsection{Nationalsozialistisches Kraftfahr-Korps (NSKK)}

Die Überlieferung aus Mittel- und Ostdeutschland und den eingegliederten Gebieten ist äußerst lückenhaft und besteht nur aus Personalunterlagen der Motorbrigade Sachsen (BA DH, 23,8 lfm, 1933-1945) und Aktensplittern Berliner und Brandenburger Einheiten (BA DH 0,6 lfm, 1931-1945, und P,Pr.Br.Rep.61) sowie der Motorgruppe Sudetenland (LT, Befehle übergeordneter Dienststellen, 1938-1944) und der Motorstandarten 203, Mährisch Schönberg (TRO, Anmeldungen 1938, Ausweise 1939-1945, Personalakten 1939-1945) und 112, Litzmannstadt (LO, 1 Bd, 1940-1941).

\subsubsection{Nationalsozialistisches Fliegerkorps (NSFK)}

Von der Gruppe 4, Berlin-Brandenburg, sind Personalunterlagen, überwiegend in Karteiform, überliefert (BA DH, 17,09 lfm), im übrigen nur Aktenreste und -splitter der Standarte 40, Teplitz-Schönau, mit Weisungen der Führung der Gruppe 6, Schlesien (1941), und Organisationsunterlagen (LT) und der Standarte 33, Jägerndorf (TRO, 3 Kts, 1938-1945) mit Personalakten und Standartenbefehlen, ferner von der Standarte 98 (AdR, Best. 08R490, 3 Kts), dabei Lehrmaterial, Reste von Schriftwechsel, Dienstanweisungen (1943-1944).

\subsubsection{Hitlerjugend $(\mathbf{H J})$}

Die nur wenigen nachweisbaren Akten stammen von der Gebietsführung Oberschlesien, Kattowitz (KAT, 13 Bde, 1939-1944, Berichte, Organisationsunterlagen, Personalakten), der Gebietsführung Sudetenland, Reichenberg (LT, 0,3 lfm, 1940-1945, Befehle und Verwaltungsanordnungen), der Gebietsführung Wien (StLAW, 1 Kt, Gebiets-, 
1938-1941, und Reichsbefehle, 1939-1941, sowie Wochensprüche, 1939-1944) und der Gebietsführung Tirol-Vorarlberg (IN, 1 Bd Disziplinar- und Strafakten, 1939-1944).

Ersatzüberlieferung liegt u. a. vor in Akten der Befehlsstelle Böhmen und Mähren der Reichsjugendführung (ZStA Prag), die Schriftwechsel (1939-1944) mit zahlreichen Gebietsführungen auch in eingegliederten Gebieten und Rundschreiben und Erlasse der Reichsjugendführung (1936-1945) enthalten, ferner in Aktensplittern von 15 oberschlesischen Bannen (KAT, dabei 20 Bde aus Kattowitz, 17 aus Hindenburg), des Banns und des BDM-Untergaus Litzmannstadt (LO, 30 Bde) und des BDM Oppeln (OP, 5 Bde, 1939-1941).

\subsubsection{NS-Frauenschaft - Gaufrauenschaftsleitung Sachsen}

ZDM MO, in Best. 519

Die Aktenreste (51 Bde, 1934-1937) betreffen Mitgliedschaft, Verhältnis zum Deutschen Frauenorden und zur Frauenorganisation des „Stahlhelm“, Reichsparteitage, Rassenpflege, Frauenarbeit in der Wirtschaft, Winterhilfswerk, Kindergärten, Anthroposophie, Kurse der DAF und Personalangelegenheiten, auch politische Überprüfungen (mit 16 Bden Personalakten).

\subsubsection{NS-Studentenbund (NSDStB)}

Lediglich Aktensplitter der Gaustudentenführung Sudetenland, Reichenberg, sind nachweisbar (LT, 0,1 lfm, 1941, 1944, u. a. Unterlagen über Ernennung von Führern 1944).

\subsubsection{NSD.-Dozentenbund}

Von der NS-Dozentenschaft Berlin sind im Universitätsarchiv die Personalunterlagen von Professoren, Privatdozenten, Lehrbeauftragten, Assistenten, Lektoren (auch Ausländern) in großem Umfang vorhanden. Vom Führer des NSD.-Dozentenbundes Jena befinden sich dort (Best. U, Abt. IV) u. a. Schriftwechsel (3 Bde, 1934-1945), auch mit Reichsstatthalter und Volksbildungsministerium (1936-1941), Berichte (19391944), Glückwünsche und Danksagungen (1938-1940), Unterlagen über das Auslandsamt (1940-1943) und über Personalangelegenheiten von Professoren, Dozenten und Assistenten (20 Bde, 1935-1945).

\subsection{Angeschlossene Verbände}

Vom Schriftgut ihrer Dienststellen und Einrichtungen in Mittel- und Ostdeutschland und den eingegliederten Gebieten blieben nur spärliche Reste erhalten, doch kann zum Teil auf Ersatzüberlieferung in Akten der zentralen Ämter verwiesen werden (vgl. Teil 1, S. 508 ff.). Keine Überlieferung konnte vom NS-Deutschen Ärztebund und vom Reichsbund der Deutschen Beamten sowie von den „betreuten“ Organisationen ermittelt werden. 


\subsubsection{Deutsche Arbeitsfront (DAF)}

Ein aus den von der DDR-Staatssicherheit gesammelten Akten gebildeter Sammelbestand (BA DH, ca. $23 \mathrm{lfm}$ ) enthält Schriftgut der Reichswaltung mit den Teilprovenienzen Führungsamt, Propagandaamt, Hauptstelle Schulung (u. a. über Lehrgänge der NS-Betriebszellenorganisation) und Büro für Arbeitsausschüsse und - vorwiegend Personalunterlagen - der Gauwaltungen Groß-Berlin (ca. 2200 Bde, u. a. Mitgliedsbücher, auch Unterlagen über Zusammenarbeit mit der Gestapo), Kurmark (u. a. betr. Vereidigung von Blockobleuten 1939), Mecklenburg (mit Sachakten und Schriftwechsel), Sachsen (mit Rundschreiben, 1936-1943), Magdeburg-Anhalt (Mitgliederkartei) und Thüringen (mit Sachakten, 1938), sowie der Schulungsburgen Lobeda und Saßnitz (Personalbögen 1938-1942).

Außerdem sind das Nachrichtenblatt der DAF-Gauwaltung Steiermark (Jg. 1-7, 1939-1945, DBL), DAF-Nachrichten der Gauwaltung Ostpreußen (1942-1943, BA KO, NSD 38) und folgende Aktenreste überliefert:

Gauwaltung Oberschlesien, Kattowitz

KAT, Best. 116/1-10 Mitgliederliste (1942), einzelne Akten (8 Bde, 1939-1945) über Organisationsfragen, Presse und Schulung (1942-1944), NS-Gemeinschaft „Kraft durch Freude“ (1942), Unterstützung für Kriegsversehrte (1940-1945), Ergänzungsüberlieferung in Akten der Kreiswaltungen Bielitz und Kattowitz (KAT, 7 bzw. 9 Bde).

Gauwaltung Sudetenland, Reichenberg

LT

Dienstweisungen und Organisationsunterlagen (5 Kts, 1939-1944), auch Unterlagen über Angelegenheiten der Bergarbeiter in Sankt Joachimsthal (1940-1943), Jahresberichte des Gauwalters (1939-1943), Lagebericht des Kreiswalters in Luditz (1945), Personalakten.

Gauwaltung Tirol-Vorarlberg, Innsbruck

IN Akten (insgesamt 26 Bde, 1938-1944) aus den Arbeitsbereichen Gaufachwalter, Arbeitseinsatz und „Kraft durch Freude“, Personal- und Disziplinarakten des Ehren- und Disziplinargerichts.

Gauwaltung Wartheland, Posen

POS, Best. 802

Allgemeine Rundschreiben und Anordnungen, Unterlagen des Gauheimstättenwerks über Bauhöfe und Umschulung der polnischen Bauarbeiter, Tätigkeitsberichte des Winterhilfswerks (1940-1941), Akten über Arbeitsdisziplin in einer Fabrik in Kalisch (Verfahren vor dem Ehren- und Disziplinargericht 1944), über Kriegswirtschaftsvergehen, polnische Beschäftigte im Handel und eine Likörfabrik (insgesamt 19 Bde, 1939-1944), Ergänzungsüberlieferung der Kreiswaltung Litzmannstadt (LO, 6 Bde, 1940-1944).

Gauwaltung Wien

StLAW

Unterlagen zu Aufgaben und Zuständigkeiten der Walter und Warte (1 Kt., 1938).

\subsubsection{NS-Volkswohlfahrt (NSV)}

Die Überlieferung aus Mittel- und Ostdeutschland und den eingegliederten Gebieten besteht lediglich aus Personalunterlagen und Fragebögen der Gauwaltung Berlin (BA DH, 9,5 lfm), Mitgliederfragebögen der Gauwaltung Mecklenburg (1935-1938, ebda), Einzelfallakten über vermittelte Adoptionen der Gauwaltung Thüringen (WE, 21 Bde), 
Organisations- und Personalunterlagen (vor allem von Kindergärtnerinnen), Einzelfallakten der Gauwaltung Oberschlesien (KAT, 13 Bde), Akten der Gauwaltung TirolVorarlberg über die Erfassung und Auflösung von Einrichtungen und Vereinen der freien Wohlfahrtspflege und die Unterbringung arbeitsunfähiger Umsiedler aus Südtirol (BA KO, Best. NS 45, 5 bzw. 3 Bde, 1938-1940 bzw. 1939-1943) und der

Gauwaltung Wartheland, Posen

POS, Best. 890

mit Rundschreiben (4 Bde, 1940-1944), internem Schriftverkehr (1939-1941), Transportlisten von balten- und wolhyniendeutschen Umsiedlern (2 Bde, 1939-1940), Listen von Wohlfahrtseinrichtungen in den Kreisen (3 Bde, 1940-1941), Schriftwechsel mit kirchlichen Einrichtungen und Wohlfahrtsvereinen (10 Bde, 1939-1940, dabei mit der jüdischen Gemeinde Posen, 1939) und Personalakten von Mitarbeitern der Verwaltung und Krankenschwestern (718 Bde), einer Mitgliederkartei (7 Bde, 1941-1944) und Anträgen auf Familienunterstützung (1941-1944) aus Litzmannstadt bei der GKW (Best. 378).

\subsubsection{NS-Kriegsopferversorgung (NSKOV)}

Überliefert sind nur Personalunterlagen unbestimmter Provenienz (BA DH, 13,9 lfm, 1933-1944), von der Gaudienststelle Sudetenland (LT) Reste von Weisungen und Rundschreiben (1940-1945) und einzelne Unterlagen im Nachlaß von Landgerichtspräsident Georg Braun, Leiter des Gauamtes für Kriegsopfer im Warthegau (POS).

\subsubsection{NS-Rechtswahrerbund}

Geringe Reste von Personalunterlagen sind von den Gauwaltungen Sachsen (DD, 1,32 lfm, 1933-1944), Tirol-Vorarlberg (IN, 1 Bd) und Wien (StLAW, 1 Mappe) überliefert.

\subsubsection{NS-Lehrerbund}

Lediglich von der Gauwaltung Sudetenland blieben (LT) 12 Geschäftsprotokolle (1938-1943) und einzelne Anordnungen und Rundschreiben (1938-1943), ferner Beurteilungen über Lehrer (1941) und Korrespondenz mit Kreiswaltungen (1939-1942) erhalten, von der Gauwaltung Tirol-Vorarlberg einige Aufnahmeanträge mit Stellungnahmen von Parteidienststellen (IN), aus Sachsen ein Aktenrest der Kreiswaltung Auerbach im Vogtland (DD, 0,25 lfm, 1933-1944).

\subsubsection{NS-Bund Deutscher Technik}

Nur vom Amt für Technik der Gauleitung Oberdonau sind einige Aktensplitter in das BA KO gelangt (Best. NS 45, 10 Bde, 1939-1945); sie betreffen Mitgliedschaft und Veranstaltungen vor allem der Fachgruppen Mechanische Technik und Energiewissenschaft im Bund. 
